\title{
Neurochondrin neurological autoimmunity
}

Shahar Shelly, MD, Thomas J. Kryzer, AS, Lars Komorowski, PhD, Ramona Miske, PhD, Mark D. Anderson, MD, Eoin P. Flanagan, MD, Shannon R. Hinson, PhD, Vanda A. Lennon, MD, PhD, Sean J. Pittock, MD, and

Andrew McKeon, MD

Neurol Neuroimmunol Neuroinflamm 2019;6:e612. doi:10.1212/NXI.0000000000000612

\section{Abstract \\ Objectives}

To describe the neurologic spectrum and treatment outcomes for neurochondrin-IgG positive cases identified serologically in the Mayo Clinic Neuroimmunology Laboratory.

\section{Methods}

Archived serum and CSF specimens previously scored positive for IgGs that stained mouse hippocampal tissue in a nonuniform synaptic pattern by immunofluorescence assay ( 89 among 616,025 screened, 1993-2019) were reevaluated. Antibody characterization experiments revealed specificity for neurochondrin, confirmed by recombinant protein assays.

\section{Results}

IgG in serum (9) or CSF (4) from 8 patients yielded identical neuron-restricted CNS patterns, most pronounced in hippocampus (stratum lucidum in particular), cerebellum (Purkinje cells and molecular layer), and amygdala. All were neurochondrin-IgG positive. Five were women; median symptom onset age was 43 years (range, 30-69). Of 7 with clinical data, 6 presented with rapidly progressive cerebellar ataxia, brainstem signs, or both; 1 had isolated unexplained psychosis 1 year prior. Five of 6 had cerebellar signs, 4 with additional brainstem symptoms or signs (eye movement abnormalities, 3; dysphagia, 2; nausea and vomiting, 1). One patient with brainstem signs (vocal cord paralysis and VII nerve palsy) had accompanying myelopathy (longitudinally extensive abnormality on MRI; aquaporin-4-IgG and myelin oligodendrocyte glycoprotein-IgG negative). The 7th patient had small fiber neuropathy only. Just 1 of 7 had contemporaneous cancer (uterine). Six patients with ataxia or brainstem signs received immunotherapy, but just 1 remained ambulatory. At last follow-up, 5 had MRI evidence of severe cerebellar atrophy.

\section{Conclusion}

In our series, neurochondrin autoimmunity was usually accompanied by a nonparaneoplastic rapidly progressive rhombencephalitis with poor neurologic outcomes. Other phenotypes and occasional paraneoplastic causes may occur.
Correspondence

Dr. McKeon

mckeon.andrew@mayo.edu 


\section{Glossary}

IFA = immunofluorescence assay; IVIg = IV immune globulin.

Autoimmune causes of movement disorders are increasingly recognized, particularly for ataxia. Identification of specific autoantibody biomarkers aids the neurologic diagnosis, expedites the search for occult cancer, and may also indicate most appropriate therapy and predict outcome. For example, Purkinje cell cytoplasmic antibody type 1 (also known as antiYo) is generally accompanied by subacute cerebellar ataxia, breast or gynecologic adenocarcinoma, and poor response to immunotherapy. ${ }^{1,2}$ In contrast, cerebellar ataxia caused by metabotropic glutamate receptor 1 (mGluR1) autoimmunity is occasionally accompanied by lymphoma, and the ataxia may respond robustly to immunotherapy. ${ }^{3}$

Herein, we describe 8 cases identified with an IgG autoantibody specific for a neuronal cytosolic protein, neurochondrin, in the course of characterizing unclassified neural-specific autoantibodies. ${ }^{4,5}$ Neurochondrin autoimmunity was previously described in 3 adult patients with cerebellar degeneration and in a child with chorea. ${ }^{6,7}$ All but 1 of the 7 patients with clinical information available in our report had predominance of rhombencephalitis (cerebellar and brainstem localization). Myelopathy and acute psychosis (accompanying ataxia) and neuropathy (without other features) were each encountered in single patients.

\section{Methods}

\section{Standard protocol approvals, registrations, and patient consents}

The Mayo Clinic Institutional Review Board approved human specimen acquisition and retrospective review of patients' histories (institutional review board \#08-006647).

\section{Study population}

Between January 1, 1993 and June 7, 2018, the Mayo Clinic Neuroimmunology Laboratory tested by tissue-based indirect immunofluorescence assay (IFA), 616,025 serum and CSF specimens submitted for service testing for autoimmune encephalitic disorders or a suspected paraneoplastic neurologic disorder. IgG in 89 of those specimens (25 CSF; 64 serum) yielded a synaptic-type hippocampal staining pattern of nonuniform focal intensity. All 89 archived specimens were retested by IFA and classified in detail according to their respective staining patterns. Eight patients with an identical staining pattern are the subject of this report; clinical information was available in 7, 5 by review of Mayo Clinic records and 2 by contact with external physicians.

\section{Laboratory methods}

Indirect IFA, dual staining by confocal microscopy, Western blots, immunoprecipitation assay, and sequencing by mass spectrometry and neurochondrin-specific cell-based assays were undertaken, as previously described (appendix e-1, links. lww.com/NXI/A143). ${ }^{8}$

\section{Results}

\section{Neural autoantigen characterization}

\section{Immunohistochemical distribution}

An identical CNS-restricted pattern of immunoreactivity (synaptic and cytoplasmic regions) was detected in 12 specimens from 8 patients ( 8 serum; 4 CSF; figure 1, A-F). IgG bound robustly to several structures: hippocampus (particularly the stratum lucidum, continuous from CA2 and CA3 regions to the dentate gyrus granular cell layer), cerebellum (Purkinje neuronal cytoplasm and diffusely in molecular layer), and amygdala. Staining was less intense in the striatum, thalamus, and cerebral cortex. Myenteric nerves had faint staining, and parenchyma of kidney and gut were nonreactive (not shown). Median endpoint dilutions were 1: 30,720 in serum (range, 1:3,840-1:61,440) and 1:64 in CSF (range, 1:2-1:256).

\section{Autoantigen identification}

Application of patient sera, but not healthy control sera, to immunoblots of mouse cerebral lysates, revealed a common immunoreactive band of approximate molecular weight 75 $\mathrm{kDa}$ (figure e-1, links.lww.com/NXI/A142). A candidate antigen was identified by protein $\mathrm{G}$ magnetic bead capture from mouse cerebral lysate using sera from patients 1, 4, and 6 (figure 1G). Mass spectrometry analysis of the electrophoretically separated proteins predicted the $75 \mathrm{kDa}$ protein to be neurochondrin.

Dual IFA staining on mouse brain sections demonstrated colocalization of patient IgGs with a commercial neurochondrin polyclonal IgG, figure 2, A-D. Sera from patients $1-6$, but not 3 healthy control sera, bound to recombinant full-length neurochondrin protein (Abcam [product number ab161548, glutathione S-transferases-tagged at $\mathrm{N}$ terminus], figure 2E). All patient specimens and 2 of 196 healthy control sera were positive by neurochondrin-specific cell-based assay; IgGs bound to neurochondrin-expressing cells, but not mock transfected cells (figure 2, F-G). Unlike the patient specimens, none of the 196 healthy control sera produced neural IgG staining by tissue-based IFA.

Summary of demographic and clinical findings Six of 8 patients were identified retrospectively from the Mayo Clinic Neuroimmunology Laboratory archives (1997-2018) and 2 patients were identified prospectively after the study 

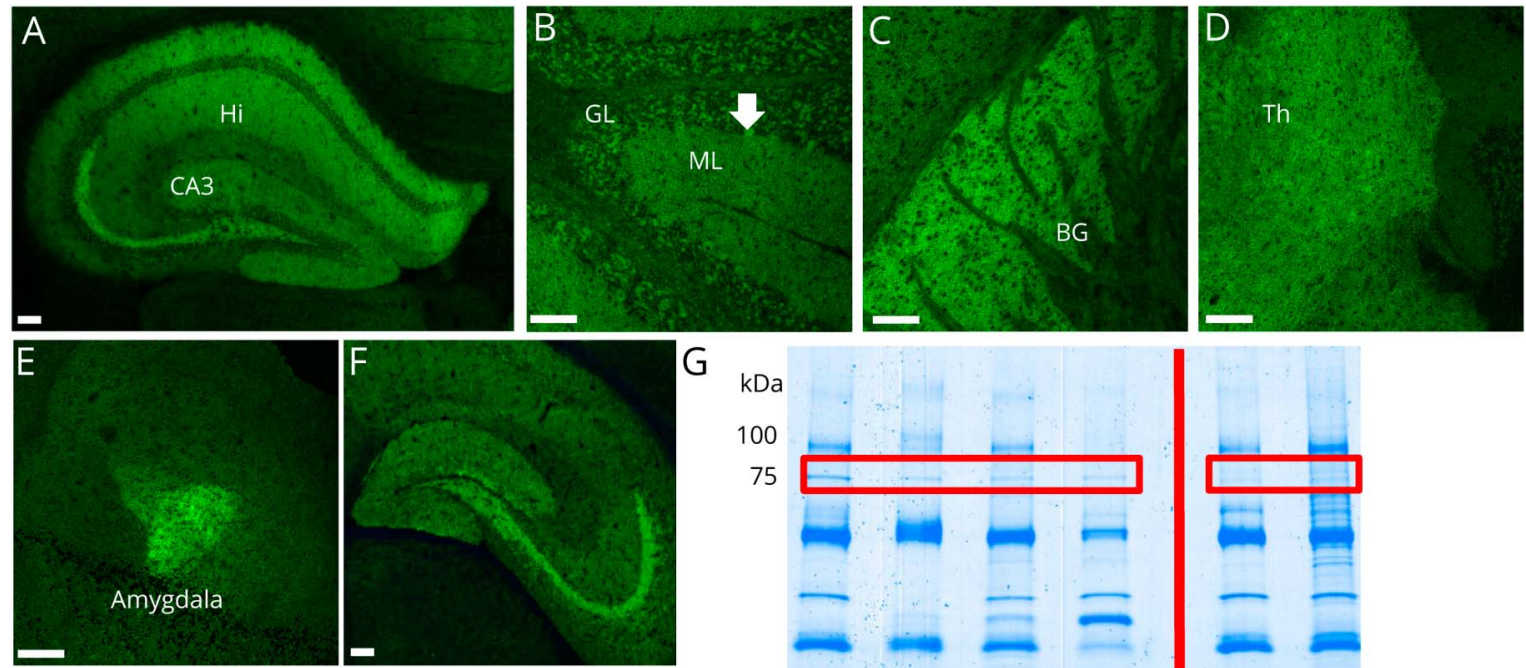

(A-F) Patient serum IgG yields a synaptic-type immunofluorescence pattern in (A) hippocampus (Hi) CA3 layer and pyramidal cells, (B) cerebellar cortex molecular layer (ML) more than granular layer (GL), and perikarya of Purkinje neurons (arrow), (C) basal ganglia (BG), (D) thalamus (Th), and (E) amygdalar neurons. (F) IgG purified from patient serum by adsorption to (and elution from) $75 \mathrm{kDa}$ Western blot band recapitulates this staining pattern (hippocampus shown). (G) Coomassie staining of mouse cerebral protein extract immunoprecipitated with serum from patients 1,4, and 6: purified IgG (from patient 1 and 4) and control serums (N1 and N2). Patient serum, but not healthy control serum, immunoprecipitated a protein of approximately $75 \mathrm{kDa}$. Scale bar $=100 \mu \mathrm{m}$. IgG = immunoglobulin G; kDa = kilodalton.

was underway. Five were female. The median symptom onset age was 43 years (range, 30-69).

\section{Neurological manifestations}

Clinical information was available for 7 of 8 patients (shown in table 1). Five of 7 were evaluated neurologically at the
Mayo Clinic. The median follow-up period was 18 months (range, 2-60). Six of 7 patients presented with a rapidly progressive rhombencephalitis (cerebellar/brainstem) over the course of a few weeks. The remaining patient had symptoms of small fiber neuropathy and dysautonomia (pain, paresthesia, and constipation) without CNS findings.

Figure 2 Confocal microscopy and recombinant protein assays (cell-based and Western blot) confirm the antigen is neurochondrin

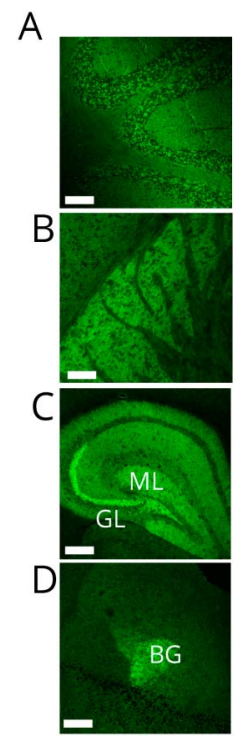

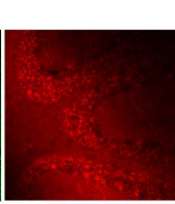

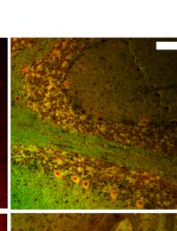

E 100
75
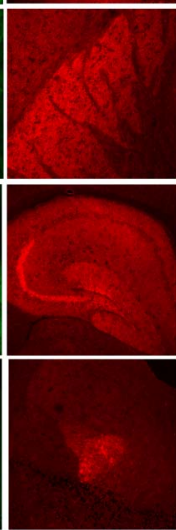

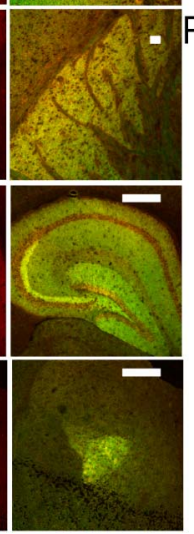
$\mathrm{KDa}$
100 75

P1 P2 P3 P4 P5 P6 N1 N2 N3

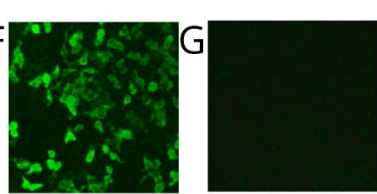


Table 1 Clinical information available for 7 neurochondrin-lgG positive patients ${ }^{a}$

\begin{tabular}{|c|c|c|c|c|c|c|c|}
\hline $\begin{array}{l}\text { Patient } \\
\text { no. sex/ } \\
\text { age }\end{array}$ & $\begin{array}{l}\text { Main neurologic } \\
\text { symptoms and signs }\end{array}$ & $\begin{array}{l}\text { Neurologic } \\
\text { diagnosis }\end{array}$ & $\begin{array}{l}\text { Other } \\
\text { diagnoses/ } \\
\text { other } \\
\text { autoantibodies } \\
\text { detected }\end{array}$ & $\begin{array}{l}\text { CSF } \\
\text { abnormalities }\end{array}$ & $\begin{array}{l}\text { Neuroimaging/ } \\
\text { electrophysiologic } \\
\text { findings }\end{array}$ & $\begin{array}{l}\text { Immuno- } \\
\text { therapy/ } \\
\text { response }\end{array}$ & $\begin{array}{l}\text { Follow-up } \\
\text { period } \\
\text { (mo)/ } \\
\text { outcome }\end{array}$ \\
\hline \multirow[t]{2}{*}{ 1.M/40 } & $\begin{array}{l}\text { Body itch for } 2 \text { wk, then } \\
\text { right LMN facial } \\
\text { weakness and } \\
\text { hypoesthesia, bilateral } \\
\text { gaze paresis, } \\
\text { dysarthria, vocal cord } \\
\text { paralysis, T8 sensory } \\
\text { level; constipation, and } \\
\text { hyperreflexia }\end{array}$ & $\begin{array}{l}\text { Brainstem } \\
\text { encephalitis } \\
\text { and thoracic } \\
\text { myelitis }\end{array}$ & $\begin{array}{l}\text { Sjögren } \\
\text { syndrome/CCP, } \\
\text { SS-A, ANA }\end{array}$ & $\begin{array}{l}\text { WBCs 185, } \\
\text { lymphocyte } \\
\text { predominant; } \\
\text { protein, 172; } \\
\text { OCB 11; IgG } \\
\text { index } 1.33\end{array}$ & $\begin{array}{l}\text { Early MRI: cerebellar } \\
\text { leptomeningeal } \\
\text { enhancement; } \\
\text { abnormal T2 signal } \\
\text { dorsal pons and } \\
\text { upper medulla. Spinal } \\
\text { MRI: long } \\
\text { intramedullary signal, } \\
\text { no enhancement. }\end{array}$ & $\begin{array}{l}\text { IV steroids; IVlg; } \\
\text { rituximab/ } \\
\text { progression }\end{array}$ & $\begin{array}{l}60 / \\
\text { wheelchair } \\
\text { bound. }\end{array}$ \\
\hline & & & & & $\begin{array}{l}\text { Late: } 5 \text { y after } \\
\text { symptom onset: } \\
\text { marked vermian } \\
\text { atrophy }\end{array}$ & & \\
\hline \multirow[t]{2}{*}{$2 . F / 33^{b}$} & $\begin{array}{l}\text { Paranoid psychosis } 1 \text { y } \\
\text { earlier; episodic ataxia } \\
\text { initially. Nystagmus, } \\
\text { cerebellar ataxia, } \\
\text { scanning dysarthria. }\end{array}$ & $\begin{array}{l}\text { Psychosis, } \\
\text { cerebellar } \\
\text { ataxia }\end{array}$ & $\begin{array}{l}\text { Hypothyroidism/ } \\
\text { SS-A and ANA }\end{array}$ & $\begin{array}{l}\text { Protein 66; OCB } \\
15\end{array}$ & $\begin{array}{l}\text { Early MRI: brain and } \\
\text { spinal cord normal }\end{array}$ & $\begin{array}{l}\text { IV steroids; IVIg/ } \\
\text { progression }\end{array}$ & $\begin{array}{l}18 / \\
\text { wheelchair } \\
\text { bound }\end{array}$ \\
\hline & & & & & $\begin{array}{l}\text { Late MRI: marked } \\
\text { cerebellar atrophy }\end{array}$ & & \\
\hline \multirow[t]{2}{*}{ 3.F/42 } & $\begin{array}{l}\text { Progressive ataxia, } \\
\text { abdominal pain and } \\
\text { vomiting, } 2 \text { mo. EOM } \\
\text { paralysis, tinnitus, } \\
\text { hearing loss, dysphagia } \\
\text { and dysarthria }\end{array}$ & $\begin{array}{l}\text { Brainstem } \\
\text { encephalitis } \\
\text { and } \\
\text { cerebellar } \\
\text { ataxia }\end{array}$ & None & $\begin{array}{l}\text { WBCs } 14, \\
\text { lymphocyte } \\
\text { predominant; } \\
\text { protein } 84 ; \\
\text { supernumerary } \\
\text { OCB; IgG index } \\
1.81\end{array}$ & $\begin{array}{l}\text { Early MRI: abnormal } \\
\text { T2 signals in midbrain, } \\
\text { pons and MCP. }\end{array}$ & $\begin{array}{l}\text { IVlg; } \\
\text { azathioprine; IV- } \\
\text { steroids }{ }^{c /} \\
\text { disease } \\
\text { progression }\end{array}$ & 27/death \\
\hline & & & & & $\begin{array}{l}\text { Late MRI: marked } \\
\text { cerebellar and } \\
\text { pontine atrophy. }\end{array}$ & & \\
\hline \multirow[t]{2}{*}{$5 . M / 35^{b}$} & $\begin{array}{l}\text { Oscillopsia, periodic } \\
\text { alternating nystagmus, } \\
\text { skew deviation; } \\
\text { progressive balance } \\
\text { difficulties; severe } \\
\text { dysmetria lower limb, } \\
\text { mild in upper limbs; } \\
\text { dysarthria }\end{array}$ & $\begin{array}{l}\text { Cerebellar } \\
\text { ataxia }\end{array}$ & $\begin{array}{l}\text { Latent TB } \\
\text { infection/ANA }\end{array}$ & Protein 77 & $\begin{array}{l}\text { Early MRI: } \\
\text { unremarkable }\end{array}$ & $\begin{array}{l}\text { IVlg; IV steroids; } \\
\text { CP; rituximab/ } \\
\text { disease } \\
\text { progression }^{d}\end{array}$ & $\begin{array}{l}10 / \\
\text { wheelchair } \\
\text { bound }\end{array}$ \\
\hline & & & & & $\begin{array}{l}\text { Late MRI: marked } \\
\text { cerebellar atrophy }\end{array}$ & & \\
\hline \multirow[t]{2}{*}{$6 . F / 53^{b}$} & $\begin{array}{l}\text { Cerebellar ataxia, left > } \\
\text { right; dysarthria, } \\
\text { vertigo, saccadic } \\
\text { pursuits, dysphagia }\end{array}$ & $\begin{array}{l}\text { Cerebellar } \\
\text { ataxia }\end{array}$ & None & $\begin{array}{l}\text { WBCs 18, } \\
\text { lymphocyte } \\
\text { predominant; } \\
\text { protein 138; IgG } \\
\text { index } 1.06\end{array}$ & $\begin{array}{l}\text { Early MRI: abnormal } \\
\text { T2 signal in left frontal } \\
\text { and cerebellar cortex }\end{array}$ & $\begin{array}{l}\text { IV steroids; } \\
\text { prolonged PO } \\
\text { steroids; } \\
\text { azathioprine/ } \\
\text { mild } \\
\text { improvement }\end{array}$ & $\begin{array}{l}\text { 48/walking } \\
\text { with cane }\end{array}$ \\
\hline & & & & & $\begin{array}{l}\text { Late MRI: cerebellar/ } \\
\text { pontine atrophy; } \\
\text { hippocampal } \\
\text { formation abnormal } \\
\text { T2 signal }\end{array}$ & & \\
\hline $7 . \mathrm{M} / 44^{\mathrm{b}}$ & $\begin{array}{l}\text { Paresthesia; } \\
\text { constipation; } \\
\text { unpleasant sensation } \\
\text { in legs, exclusively } \\
\text { present or worse } \\
\text { during periods of rest } \\
\text { or inactivity such as } \\
\text { lying or sitting; normal } \\
\text { examination }\end{array}$ & $\begin{array}{l}\text { Small fiber } \\
\text { neuropathy }\end{array}$ & $\begin{array}{l}\text { ANA (RNP- } \\
\text { specific) }\end{array}$ & Not tested & $\begin{array}{l}\text { MRI: brain and } \\
\text { cervical, thoracic, and } \\
\text { lumbar spine are } \\
\text { normal }\end{array}$ & $\begin{array}{l}\text { No } \\
\text { immunotherapy } \\
\text { given/stable } \\
\text { disease no } \\
\text { progression }\end{array}$ & $\begin{array}{l}\text { 2/normal } \\
\text { gait }\end{array}$ \\
\hline
\end{tabular}


Table 1 Clinical information available for 7 neurochondrin-lgG positive patients ${ }^{\mathrm{a}}$ (continued)

\begin{tabular}{llllll}
\hline $\begin{array}{l}\text { Patient } \\
\text { no. sex/ } \\
\text { age }\end{array}$ & $\begin{array}{l}\text { Main neurologic } \\
\text { symptoms and signs }\end{array}$ & $\begin{array}{l}\text { Neurologic } \\
\text { diagnosis }\end{array}$ & $\begin{array}{l}\text { Other } \\
\text { diagnoses/ } \\
\text { other } \\
\text { autoantibodies } \\
\text { detected }\end{array}$ & $\begin{array}{l}\text { CSF } \\
\text { abnormalities }\end{array}$ & $\begin{array}{l}\text { Follow-up } \\
\text { period } \\
\text { electrophysiologic } \\
\text { findings } \\
\text { outcome }\end{array}$ \\
\hline $\mathbf{8 . F / 6 9}$ & $\begin{array}{l}\text { Nausea, vomiting, } \\
\text { nystagmus, } \\
\text { incoordination }\end{array}$ & $\begin{array}{l}\text { Cerebellar } \\
\text { ataxia }\end{array}$ & $\begin{array}{l}\text { Uterine } \\
\text { carcinoma, } \\
\text { poorly } \\
\text { differentiated }\end{array}$ & NA & Early MRI: bilateral \\
response \\
striatal and \\
hippocampal T2 \\
abnormalities
\end{tabular}

Abbreviations: $A N A=$ antinuclear antibody; $C P=$ cyclophosphamide; $E O M=$ extra ocular muscle; $F=$ female; IgG = immunoglobulin G; IVIg = IV immune globulin; $\mathrm{M}=$ male; $\mathrm{MCP}=$ middle cerebellar peduncle; $\mathrm{NA}=$ not available; $\mathrm{NCS}$ = nerve conduction study; OCB = oligoclonal bands; $\mathrm{PO}=$ per os; RNP = ribonucleoprotein; RLS = restless leg syndrome; TB = tuberculosis; WBC = white blood cells.

CSF normal ranges: protein $\leq 35 \mathrm{mg} / \mathrm{dL}$; leukocyte count $<5 / \mathrm{mL}$; CSF-unique OCB $<4$; IgG index $\leq 0.85$.

${ }^{a}$ The 8th patient identified serologically (patient 4) had no clinical information available.

${ }^{b}$ Five patients were evaluated at Mayo Clinic. Negative testing for alternative causes of ataxia included: vitamin B12, 6; vitamin E, 4; copper, 4; ceruloplasmin, 3.

c Immunotherapy was stopped 6 months prior to death.

dImmunotherapy was started, 1 year after symptoms started.

Among the 6 patients with CNS disorders, 5 had cerebellar signs including gait difficulties, dysarthria, and prominent dysmetria. One of those 5 patients had an isolated episode of unexplained psychosis 1 year prior to the onset of ataxia. She developed an unusual self-resolving paranoid state without a prior history of drug abuse or psychiatric illness. Brainstem symptoms or signs, present in 4 of those 5 patients, included eye movement abnormalities in all, dysphagia in 2 , and nausea and vomiting in 1 . The 6 th patient, without cerebellar signs, had brainstem signs, vocal cord paralysis, and unilateral VIIth cranial nerve palsy accompanying myelopathy (longitudinally extensive T2 signal abnormality on MRI, though aquaporin-4IgG and myelin oligodendrocyte glycoprotein-IgG serum tests were negative). The myelopathy was preceded by diffuse migratory itch 2 weeks prior to the onset of weakness.

\section{Oncologic and autoimmune accompaniments}

Of the 7 patients with records available, 1 had poorly differentiated uterine carcinoma contemporaneous with neurologic symptom onset. Five others had cancer-negative whole-body imaging (PET-CT in 4 and CT alone in 1). Two patients had coexisting autoimmune disease (Sjögren syndrome, 1; thyroiditis, 1).

\section{CSF studies}

CSF findings were abnormal in all 5 patients for whom data were available. All had signs of inflammation (increases in protein [all; median value, $80.5 \mathrm{mg} / \mathrm{dL}$; range, 49-172], leukocyte count [3; all lymphocyte predominant, median value, $18 / \mu \mathrm{L}$; range, $14-185]$; IgG index and synthesis rate [3], or CSF-exclusive oligoclonal bands [3], table 1).

\section{Imaging}

Brain MRI images from early in the disease course were available for review in all patients with cerebellar or brainstem symptoms and signs, with the exception of patient 8 (figure 3 ). These early images were normal in 2 patients, demonstrated T2 abnormalities in 3 (cerebellum [2], brainstem [2], and frontal lobe [1]), and rhombencephalon-restricted postgadolinium abnormalities in 1, figure $3, \mathrm{~A}-\mathrm{E}$, and $\mathrm{H}$. The patient with rhombencephalomyelitis, and cerebellar leptomeningeal enhancement, also had nonenhancing T2 abnormalities of the central thoracic cord, figure 3, F and G. Brain MRI acquired late in the disease course in 5 patients revealed severe cerebellar atrophy in all, figure 3, I-M. Patients 6 and 8, with ataxic neurologic phenotypes only, had bilateral hippocampal T2 signal abnormalities (image available for patient 6 , figure $3, \mathrm{~N}$ ). Thermoregulatory sweat test in the patient with small fiber neuropathy demonstrated hypohidrosis of the distal lower extremities.

\section{Treatment and outcomes}

Five of 6 patients with an ataxic or brainstem syndrome were treated with immunotherapy within 1 month of symptom onset (corticosteroids, 5; IV immune globulin [IVIg], 3; plasma exchange, 1). Despite early intervention, only 1 patient (patient 6) remained ambulatory; she had received corticosteroids. Three patients became wheelchair-bound, and 1 died from complications of neurologic progression. One patient who commenced treatment 1 year after symptom onset remained wheelchair-bound. It was the opinion of the prescribing physicians that some immune therapies (rituximab, azathioprine, IVIg) slowed the rate of disease progression (3) or mildly improved symptoms (patient 6 who received both IV and oral corticosteroids, and azathioprine, table 1).

\section{Discussion}

We report 8 patients in whom we identified neurochondrin IgG incidentally in the course of characterizing an unclassified neural-specific IgG autoantibody. Specificity was confirmed by affinity purification of the antigen, mass spectrometry, confocal microscopy and protein-specific assays (Western blot and transfected cell-based).

Neurochondrin is a leucine-rich protein expressed in brain, bone (osteoblasts and osteoclasts), and cartilage., ${ }^{5,9}$ It 
Figure $3 \mathrm{MRI}$ images of patients seropositive for neurochondrin-IgG acquired early (A-H) and late (I-M) in the disease course
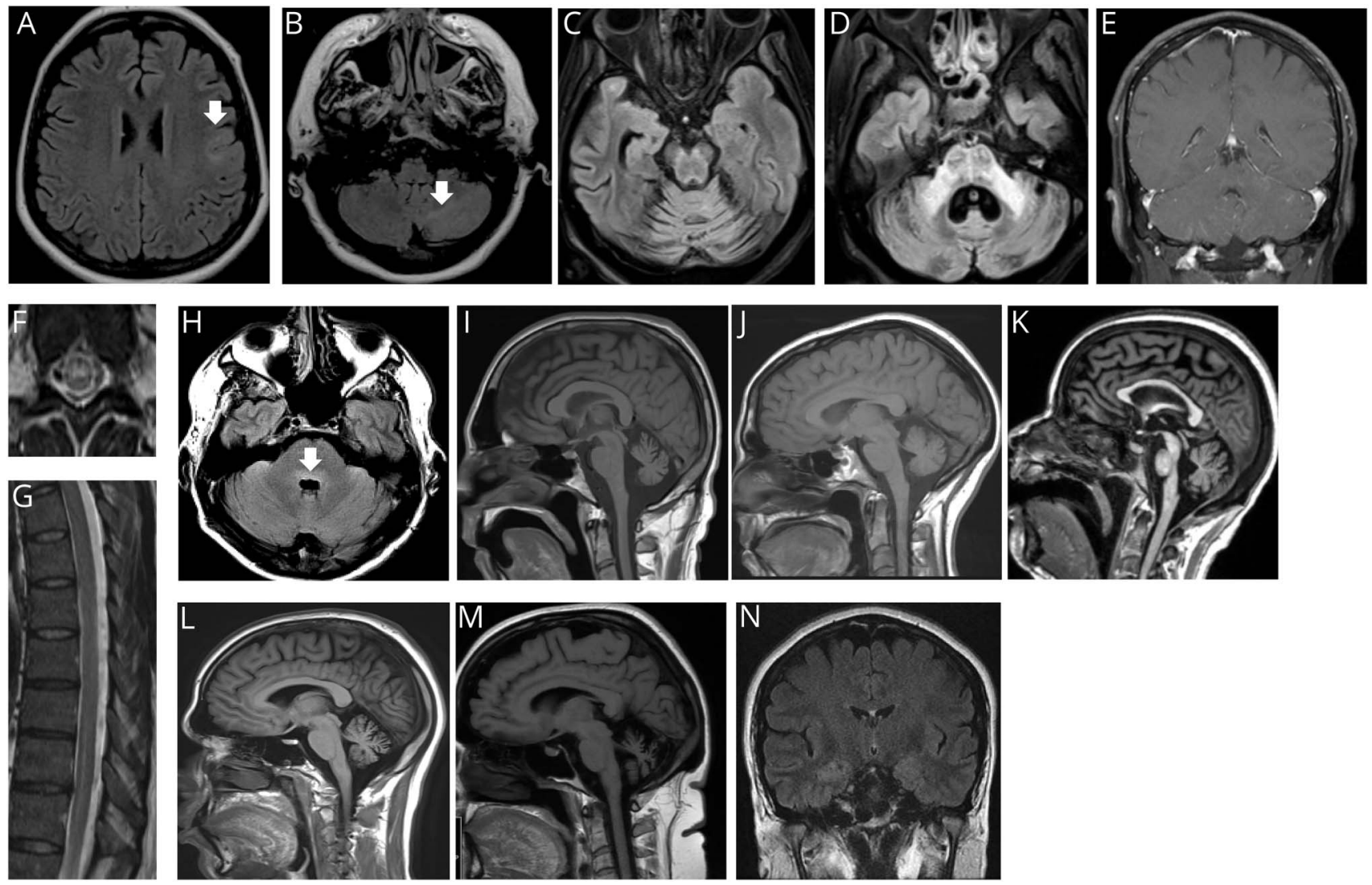

(A) Patient 6. T2 FLAIR axial of brain demonstrates T2 signal abnormality in gray-white matter junction of the left frontal lobe (arrow). (B) Patient 6. T2 FLAIR axial of brain demonstrates left cerebellar T2 hyperintensity (arrow). (C-D) Patient 3. FLAIR axial of brain demonstrates T2 hyperintensities, pancerebellar pons, and in middle cerebellar peduncles. (E) Patient 1. T1 postgadolinium, sagittal of brain demonstrates cerebellar leptomeningeal enhancement. (F-G) Patient 1. T2 axial and sagittal thoracic of spine demonstrate intramedullary (F) and longitudinal hyperintensities (G). (H) Patient 1. T2 FLAIR axial of brain demonstrates dorsal pons hyperintensity (arrow). (I-M) Patients 1, 2, 3, 5, and 6. T1 sagittal of brain demonstrates cerebellar atrophy. (N) Patient 6. T2 FLAIR coronal of brain also had bilateral hippocampal T2 abnormalities at follow-up. FLAIR = fluid attenuated inversion recovery.

enhances cell-surface localization of certain membrane-bound proteins, such as metabotropic glutamate receptors, but is itself a cytoplasmic antigen. ${ }^{5}$ While reliable as a biomarker of neurologic autoimmunity, neurochondrin-IgG is not pathogenic, but likely a proxy for a cytotoxic $\mathrm{T}$ cell-mediated pathogenesis. Consistent with our clinical findings, neurochondrin is highly expressed in cerebellar Purkinje cells, the brainstem, the lateral parts of the central amygdalar nuclei, the hippocampal pyramidal cells, and also the autonomic and peripheral nervous systems. ${ }^{4}$ Relevant to the psychosis, myelopathy, and neuropathy phenotypes, respectively, neurochondrin knockout mice have a schizophrenia-like behavioral phenotype, neurochondrin is required for the correct localization of a survival motor neuron protein, and is expressed in rat peripheral nerve. ${ }^{5,10,11}$ In bone, neurochondrin inhibits osteoclast- and macrophage-induced hydroxyapatite bone resorption. ${ }^{12}$ None of our patients was known to have had metabolic bone disease.

We confirm the principal clinical accompaniments originally described, namely, severe cerebellar ataxia and other features of rhombencephalitis, and usually without evidence of systemic cancer. Other phenotypes include chorea ${ }^{7}$ and small fiber neuropathy (reported herein). Two of our patients with rhombencephalitis had additional neuropsychiatric findings (psychosis or myelopathy). Neuroradiological findings were diverse, though were cerebellum and brainstem predominant. The diversity of cerebellar and extracerebellar T2 abnormalities, not typical for other autoimmune ataxias, could serve as a clue to neurochondrin CNS autoimmunity.

Neurochondrin IgG is one of several reported biomarkers of autoimmune ataxias with (usually) nonparaneoplastic, idiopathic autoimmune cause. ${ }^{13-15}$ These are listed in order of reported commonality in table 2 .

The almost universal lack of neurologic improvements from immunotherapies in neurochondrin autoimmunity is consistent with other nonantibody-mediated, intracellular antigen-defined autoimmune ataxias, for some of which $\mathrm{CD}^{+}$cytotoxic $\mathrm{T}$-cell infiltrative immunopathology has been demonstrated. ${ }^{6,16,17}$ We identified 1 patient who remained 
Table 2 Autoimmune (usually nonparaneoplastic) cerebellar/brainstem ataxias

\begin{tabular}{|c|c|c|c|c|}
\hline $\begin{array}{l}\text { Antibody (references: } \\
\text { first report, largest } \\
\text { series) }\end{array}$ & $\begin{array}{l}\text { Neural } \\
\text { localization } \\
\text { of antigen }\end{array}$ & $\begin{array}{l}\text { No. of patients } \\
\text { with ataxia } \\
\text { reported }\end{array}$ & Neurological features & $\begin{array}{l}\text { Occasional cancer association, } \\
\text { immune treatment response }\end{array}$ \\
\hline GAD65 18,19 & Cytoplasm & $>50$ & $\begin{array}{l}\text { Ataxia (gait only or pancerebellar); diplopia, vertigo, } \\
\text { oscillopsia }\end{array}$ & $\begin{array}{l}\text { Cancers rare, diverse; a minority } \\
\text { improve }\end{array}$ \\
\hline mGluR1 3,20 & Cell surface & 14 & Pancerebellar ataxia; 1 case of encephalitis & Hodgkin lymphoma; responsive \\
\hline Neurochondrin ${ }^{6}$ & Cytoplasm & 14 (7 herein) & $\begin{array}{l}\text { Ataxia, brainstem encephalitis, chorea, myelitis, } \\
\text { neuropathy }\end{array}$ & $\begin{array}{l}1 \text { case of uterine carcinoma; } \\
\text { infrequent improvement }\end{array}$ \\
\hline$D_{P P X}{ }^{21,} 22$ & Cell surface & 14 & $\begin{array}{l}\text { Ataxia and brainstem symptoms common, though } \\
\text { symptoms are diverse and often multifocal }\end{array}$ & $\begin{array}{l}\text { B-cell neoplasia, rare; responsive } \\
\text { but chronic-relapsing in many }\end{array}$ \\
\hline GluRס2/GluD2 $2^{23,24}$ & Cell surface & 11 & $\begin{array}{l}\text { Ataxia, opsoclonus-myoclonus ataxia syndrome, } \\
\text { encephalitis; children (ataxia) and adults } \\
\text { (encephalitis) reported }\end{array}$ & $\begin{array}{l}\text { No cancer association; } \\
\text { responsive }\end{array}$ \\
\hline AP3B2 (anti-Nb) $)^{25,} 26$ & Cytoplasm & 10 & $\begin{array}{l}\text { Ataxia (cerebellar, dorsal column disease, or } \\
\text { sensory ganglionopathy) }\end{array}$ & $\begin{array}{l}1 \text { case of renal cell carcinoma; } \\
\text { may stabilize but do not improve }\end{array}$ \\
\hline ITPR-1 ${ }^{27,28}$ & Cytoplasm & 9 & $\begin{array}{l}\text { Ataxia (usual), seizures, myelopathy, neuropathy } \\
\text { (rare) }\end{array}$ & $\begin{array}{l}\text { Breast adenocarcinoma, non- } \\
\text { small cell lung carcinoma; no } \\
\text { improvements }\end{array}$ \\
\hline ARHGAP26 (anti-Ca) $)^{29}$ & Cytoplasm & 6 & $\begin{array}{l}\text { Ataxia (usual), cognitive impairment and psychosis } \\
\text { (rare) }\end{array}$ & $\begin{array}{l}1 \text { case of ovarian } \\
\text { adenocarcinoma; modest short- } \\
\text { lived responses }\end{array}$ \\
\hline Septin- $5^{8}$ & Cell surface & 5 & $\begin{array}{l}\text { Pancerebellar ataxia; oscillopsia and vertigo } \\
\text { prominent }\end{array}$ & $\begin{array}{l}\text { No cancer association; } \\
\text { responsive }\end{array}$ \\
\hline IgLON5 ${ }^{30,31}$ & Cytoplasm & 4 & $\begin{array}{l}\text { Prominent sleep disorders; can have ataxia, chorea } \\
\text { or Parkinsonism }\end{array}$ & Usually not treatment responsive \\
\hline Homer-3 $3^{32}$ & Cytoplasm & 3 & Ataxia & $\begin{array}{l}\text { No cancer association; partial } \\
\text { response reported }\end{array}$ \\
\hline
\end{tabular}

Abbreviations: AP3B2 = adaptor protein 3B2; ARHGAP26 = RhoGTPase-activating protein 26; DPPX = dipeptidyl peptidase-like 6; GAD65 = glutamic acid decarboxylase, $65 \mathrm{kDa}$ isoform; GluRD2 = glutamate receptor delta 2; IgLON5 = immunoglobulin-like molecule 5; ITPR1 = inositol triphosphate receptor-1; mGluR1 = metabotropic glutamate receptor 1.

ambulatory after corticosteroid therapy and did not progress after treatment with azathioprine. It is possible that future patients treated early with high-dose steroids and $\mathrm{T}$ celldirected therapy (cyclophosphamide) might have better outcomes. The most robust responses to immune therapies have been reported among autoimmune ataxia patients harboring antibodies reactive with cell surface antigens (table 2). ${ }^{16}$

For patients presenting with unexplained ataxia or brainstem symptoms and signs of subacute onset, comprehensive neural IgG screening with tissue-based immunohistochemistry permits simultaneous evaluation for a growing list of neuralspecific IgGs, including neurochondrin-IgG.

\section{Study funding}

This study was funded by the Mayo Clinic Center for Individualized Medicine and Euroimmun.

\section{Disclosure}

S. Shelly reports no disclosures. T.J. Kryzer has a financial interest in the following intellectual property: "Marker for Neuromyelitis Optica." A patent has been issued for this technology, and it has been licensed to commercial entities. They have received cumulative royalties of greater than the federal threshold for significant financial interest from the licensing of these technologies, but receive no royalties from the sale of these tests by Mayo Medical Laboratories. L. Komorowski is employed by Euroimmun AG. R. Miske is employed by Euroimmun AG. M.D. Anderson reports no disclosures. E. Flanagan is a site principal investigator in a randomized placebo-controlled clinical trial of Inebilizumab (A CD19 inhibitor) in neuromyelitis optica spectrum disorders funded by MedImmune/Viela Bio and has served on its advisory board. S.R. Hinson reports no disclosures; V.A. Lennon has a patent application pending for septin-5 IgG as a biomarker of autoimmune neurologic disease. She has a financial interest in the following intellectual property: "Marker for Neuromyelitis Optica." A patent has been issued for this technology, and it has been licensed to commercial entities. They have received cumulative royalties of greater than the federal threshold for significant financial interest from the licensing of these technologies, but receive no royalties from the sale of these tests by Mayo Medical Laboratories. S.J. Pittock has a patent application pending for septin-5 IgG as a biomarker of autoimmune neurologic disease, and holds 
patents that relate to functional $\mathrm{AQP} 4 / \mathrm{NMO}-\mathrm{IgG}$ assays and NMO-IgG as a cancer marker; consulted for Alexion and Medimmune; and received research support from Grifols, Medimmune, and Alexion. All compensation for consulting activities is paid directly to Mayo Clinic. A. McKeon has received research funding from Alexion, Grifols, and Medimmune, and has patents pending for the following IgGs as biomarkers of autoimmune neurologic disorders (septin-5, Kelch-like protein 11, GFAP, PDE10A, and MAP1B). Go to Neurology.org/NN for full disclosures.

\section{Publication history}

Received by Neurology: Neuroimmunology \& Neuroinflammation July 1, 2019. Accepted in final form July 30, 2019.

Appendix Authors

\begin{tabular}{lll}
\hline Name & Location & Contribution \\
\hline $\begin{array}{l}\text { Shahar } \\
\text { Shelly, MD }\end{array}$ & Mayo Clinic & $\begin{array}{l}\text { Data collection, analysis, and } \\
\text { interpretation, and drafting of } \\
\text { manuscript }\end{array}$ \\
\hline $\begin{array}{l}\text { Thomas J. } \\
\text { Kryzer, AS }\end{array}$ & Mayo Clinic & $\begin{array}{l}\text { Data collection, analysis, and } \\
\text { interpretation, and critical revision of } \\
\text { manuscript }\end{array}$ \\
\hline $\begin{array}{l}\text { Lars } \\
\text { Komorowski, } \\
\text { PhD }\end{array}$ & AG & $\begin{array}{l}\text { Data collection, analysis, and } \\
\text { interpretation, and critical revision of } \\
\text { manuscript }\end{array}$ \\
\hline $\begin{array}{l}\text { Ramona } \\
\text { Miske, PhD }\end{array}$ & EG & $\begin{array}{l}\text { Data collection, analysis, and } \\
\text { interpretation, and critical revision of } \\
\text { manuscript }\end{array}$ \\
\hline
\end{tabular}

\begin{tabular}{lll}
\hline $\begin{array}{l}\text { Mark D. } \\
\text { Anderson, }\end{array}$ & University of & $\begin{array}{l}\text { Data collection, analysis, and } \\
\text { MD }\end{array}$ \\
Mississippi & $\begin{array}{l}\text { interpretation, and critical revision of } \\
\text { manuscript }\end{array}$
\end{tabular}

\begin{tabular}{lll}
\hline Eoin P. & Mayo Clinic & $\begin{array}{l}\text { Data collection, analysis, and } \\
\text { interpretation, and critical revision of } \\
\text { manuscript }\end{array}$
\end{tabular}

\begin{tabular}{ll}
\hline Shannon R. Mayo Clinic & $\begin{array}{l}\text { Data collection, analysis, and } \\
\text { interpretation, and critical revision of } \\
\text { Hinson, PhD }\end{array}$
\end{tabular}

\begin{tabular}{|c|c|c|}
\hline $\begin{array}{l}\text { Vana A. } \\
\text { Lennon, MD, } \\
\text { PhD }\end{array}$ & Mayo Clinic & $\begin{array}{l}\text { Data collection, analysis, and } \\
\text { interpretation, and critical revision of } \\
\text { manuscript }\end{array}$ \\
\hline
\end{tabular}

Sean J. Mayo Clinic Data collection, analysis, and

Sean J. Mayo Clinic Data collection, analysis, and

Pittock, MD interpretation, and critical revision of
manuscript

\begin{tabular}{lll}
\hline Andrew & Mayo Clinic & Study concept and design; data \\
Mckeon, MD & & $\begin{array}{l}\text { collection, analysis, and interpretation; } \\
\text { drafting and critical revision of } \\
\text { manuscript; and study supervision }\end{array}$
\end{tabular}

\section{References}

1. Peterson K, Rosenblum MK, Kotanides H, Posner JB. Paraneoplastic cerebellar degeneration. I. A clinical analysis of 55 anti-Yo antibody-positive patients. Neurology 1992;42:1931-1937.

2. McKeon A, Tracy JA, Pittock SJ, Parisi JE, Klein CJ, Lennon VA. Purkinje cell cytoplasmic autoantibody type 1 accompaniments: the cerebellum and beyond. Arch Neurol 2011;68:1282-1289.

3. Lopez-Chiriboga AS, Komorowski L, Kumpfel T, et al. Metabotropic glutamate receptor type 1 autoimmunity: clinical features and treatment outcomes. Neurology 2016;86:1009-1013.
4. Istvanffy R, Vogt Weisenhorn DM, Floss T, Wurst W. Expression of neurochondrin in the developing and adult mouse brain. Dev Genes Evol 2004;214:206-209.

5. Wang H, Westin L, Nong Y, et al. Norbin is an endogenous regulator of metabotropic glutamate receptor 5 signaling. Science 2009;326:1554-1557.

6. Miske R, Gross CC, Scharf M, et al. Neurochondrin is a neuronal target antigen in autoimmune cerebellar degeneration. Neurol Neuroimmunol Neuroinflamm 2017;4 e307.

7. Rommel FR, Miske R, Stocker W, Arneth B, Neubauer BA, Hahn A. Chorea minor associated with anti-neurochondrin autoantibodies. Neuropediatrics 2017;48: 482-483.

8. Honorat JA, Lopez-Chiriboga AS, Kryzer TJ, et al. Autoimmune septin- 5 cerebellar ataxia. Neurol Neuroimmunol Neuroinflamm 2018;5:e474.

9. Dateki M, Horii T, Kasuya Y, et al. Neurochondrin negatively regulates CaMKII phosphorylation, and nervous system-specific gene disruption results in epileptic seizure. J Biol Chem 2005;280:20503-20508.

10. Thompson LW, Morrison KD, Shirran SL, et al. Neurochondrin interacts with the SMN protein suggesting a novel mechanism for spinal muscular atrophy pathology. J Cell Sci 2018;131:jcs211482.

11. Shinozaki K, Kume H, Kuzume H, Obata K, Maruyama K. Norbin, a neuriteoutgrowth-related protein, is a cytosolic protein localized in the somatodendritic region of neurons and distributed prominently in dendritic outgrowth in Purkinje cells. Brain Res Mol Brain Res 1999;71:364-368.

12. Ishiduka $Y$, Mochizuki R, Yanai $K$, et al. Induction of hydroxyapatite resorptive activity in bone marrow cell populations resistant to bafilomycin A1 by a factor with restricted expression to bone and brain, neurochondrin. Biochim Biophys Acta 1999;1450: 92-98.

13. Jarius S, Wildemann B. "Medusa head ataxia": the expanding spectrum of Purkinje cell antibodies in autoimmune cerebellar ataxia. Part 3: anti-Yo/CDR2, anti-Nb/AP3B2, PCA-2, anti-Tr/DNER, other antibodies, diagnostic pitfalls, summary and outlook. J Neuroinflamm 2015;12:168.

14. Jarius S, Wildemann B. "Medusa head ataxia": the expanding spectrum of Purkinje cell antibodies in autoimmune cerebellar ataxia. Part 2: anti-PKC-gamma, anti-GluRdelta2, anti-Ca/ARHGAP26 and anti-VGCC. J Neuroinflamm 2015;12:167.

15. Jarius S, Wildemann B. "Medusa-head ataxia": the expanding spectrum of Purkinje cell antibodies in autoimmune cerebellar ataxia. Part 1: anti-mGluR1, anti-Homer-3, antiSj/ITPR1 and anti-CARP VIII. J Neuroinflamm 2015;12:166.

16. Jones AL, Flanagan EP, Pittock SJ, et al. Responses to and outcomes of treatment of autoimmune cerebellar ataxia in adults. JAMA Neurol 2015;72:1304-1312.

17. Albert ML, Darnell JC, Bender A, Francisco LM, Bhardwaj N, Darnell RB. Tumorspecific killer cells in paraneoplastic cerebellar degeneration. Nat Med 1998;4: 1321-1324.

18. Saiz A, Arpa J, Sagasta A, et al. Autoantibodies to glutamic acid decarboxylase in three patients with cerebellar ataxia, late-onset insulin-dependent diabetes mellitus, and polyendocrine autoimmunity. Neurology 1997;49:1026-1030.

19. Pittock SJ, Yoshikawa H, Ahlskog JE, et al. Glutamic acid decarboxylase autoimmunity with brainstem, extrapyramidal, and spinal cord dysfunction. Mayo Clin Proc 2006; 81:1207-1214.

20. Sillevis Smitt P, Kinoshita A, De Leeuw B, et al. Paraneoplastic cerebellar ataxia due to autoantibodies against a glutamate receptor. N Engl J Med 2000;342:21-27.

21. Boronat A, Gelfand JM, Gresa-Arribas N, et al. Encephalitis and antibodies to dipeptidyl-peptidase-like protein-6, a subunit of Kv4.2 potassium channels. Ann Neurol 2013;73:120-128.

22. Tobin WO, Lennon VA, Komorowski L, et al. DPPX potassium channel antibody: frequency, clinical accompaniments, and outcomes in 20 patients. Neurology 2014; 83:1797-1803.

23. Sugiyama N, Hamano S, Mochizuki M, Tanaka M, Takahashi Y. A case of chronic cerebellitis with anti-glutamate receptor delta 2 antibody [in Japanese]. No To Hattatsu 2004;36:60-63.

24. Berridge G, Menassa DA, Moloney T, et al. Glutamate receptor delta2 serum antibodies in pediatric opsoclonus myoclonus ataxia syndrome. Neurology 2018;91: e714-e723.

25. Darnell RB, Furneaux HM, Posner JB. Antiserum from a patient with cerebellar degeneration identifies a novel protein in Purkinje cells, cortical neurons, and neuroectodermal tumors. J Neurosci 1991;11:1224-1230.

26. Honorat JA, Lopez-Chiriboga, Kryzer TJ, et al. Autoimmune gait disturbance accompanying adaptor protein-3B2-IgG. Neurology 2019;93:1-10.

27. Jarius S, Scharf M, Begemann N, et al. Antibodies to the inositol 1,4,5-trisphosphate receptor type 1 (ITPR1) in cerebellar ataxia. J Neuroinflamm 2014;11:206.

28. Alfugham N, Gadoth A, Lennon VA, et al. ITPR1 autoimmunity: frequency, neurologic phenotype, and cancer association. Neurol Neuroimmunol Neuroinflamm 2018; 5:e418.

29. Jarius S, Wandinger KP, Horn S, Heuer H, Wildemann B. A new Purkinje cell antibody (anti-Ca) associated with subacute cerebellar ataxia: immunological characterization. J Neuroinflamm 2010;7:21.

30. Sabater L, Gaig C, Gelpi E, et al. A novel non-rapid-eye movement and rapid-eyemovement parasomnia with sleep breathing disorder associated with antibodies to IgLON5: a case series, characterisation of the antigen, and post-mortem study. Lancet Neurol 2014;13:575-586

31. Gaig C, Graus F, Compta Y, et al. Clinical manifestations of the anti-IgLON5 disease. Neurology 2017;88:1736-1743.

32. Zuliani L, Sabater L, Saiz A, Baiges JJ, Giometto B, Graus F. Homer 3 autoimmunity in subacute idiopathic cerebellar ataxia. Neurology 2007;68:239-240. 


\section{Neurology \\ Neuroimmunology \& Neuroinflammation}

Neurochondrin neurological autoimmunity

Shahar Shelly, Thomas J. Kryzer, Lars Komorowski, et al.

Neurol Neuroimmunol Neuroinflamm 2019;6;

DOI 10.1212/NXI.0000000000000612

This information is current as of September 11, 2019

Updated Information \&

Services

References

Subspecialty Collections

Permissions \& Licensing

Reprints including high resolution figures, can be found at:

http://nn.neurology.org/content/6/6/e612.full.html

This article cites 32 articles, 4 of which you can access for free at: http://nn.neurology.org/content/6/6/e612.full.html\#\#ref-list-1

This article, along with others on similar topics, appears in the following collection(s):

All Movement Disorders

http://nn.neurology.org//cgi/collection/all_movement_disorders

Autoimmune diseases

http://nn.neurology.org//cgi/collection/autoimmune_diseases

Gait disorders/ataxia

http://nn.neurology.org//cgi/collection/gait_disorders_ataxia

Information about reproducing this article in parts (figures,tables) or in its entirety can be found online at:

http://nn.neurology.org/misc/about.xhtml\#permissions

Information about ordering reprints can be found online:

http://nn.neurology.org/misc/addir.xhtml\#reprintsus

Neurol Neuroimmunol Neuroinflamm is an official journal of the American Academy of Neurology.

Published since April 2014, it is an open-access, online-only, continuous publication journal. Copyright

Copyright (C) 2019 The Author(s). Published by Wolters Kluwer Health, Inc. on behalf of the American

Academy of Neurology.. All rights reserved. Online ISSN: 2332-7812.

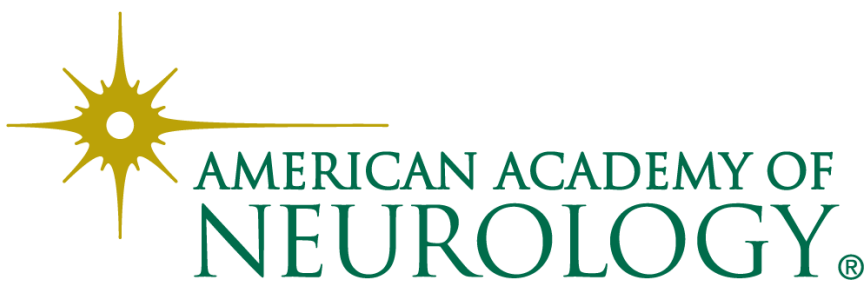

